\title{
Transcription factors and evolution: An integral part of gene expression (Review)
}

\author{
THANASIS MITSIS ${ }^{1}$, ASPASIA EFTHIMIADOU ${ }^{2}$, FLORA BACOPOULOU ${ }^{3}$, \\ DIMITRIOS VLACHAKIS ${ }^{1,4,5}$, GEORGE P. CHROUSOS $^{3,4}$ and ELIAS ELIOPOULOS ${ }^{1}$
}

\begin{abstract}
${ }^{1}$ Genetics and Computational Biology Group, Laboratory of Genetics, Department of Biotechnology, Agricultural University of Athens, 11855 Athens; ${ }^{2}$ Hellenic Agricultural Organization-Demeter, Institute of Soil and Water Resources, Department of Soil Science of Athens, 13561 Athens; ${ }^{3}$ Division of Endocrinology, Metabolism and Diabetes, First Department of Pediatrics, National and Kapodistrian University of Athens Medical School, 'Aghia Sophia' Children's Hospital,

11527 Athens; ${ }^{4}$ Division of Endocrinology and Metabolism, Center of Clinical, Experimental Surgery and Translational Research, Biomedical Research Foundation of the Academy of Athens, 11527 Athens, Greece; ${ }^{5}$ Department of Informatics, Faculty of Natural and Mathematical Sciences, King's College London, London WC2R 2LS, UK
\end{abstract}

Received December 10, 2019; Accepted January 16, 2020

DOI: $10.3892 /$ wasj.2020.32

\begin{abstract}
Gene expression is a complex process that is essential to living organisms. Gene expression plays the important role of converting information that is encoded in a gene into a functional product. The primary regulators of gene expression are transcription factors (TFs). TFs are proteins that can bind specific DNA sequences and regulate gene expression. Their evolution is influenced by a large number of factors, including epigenetic mechanisms, gene regulatory elements and molecular cofactors. These molecular mediators, along with transcription factors, form a network that governs gene expression. Elucidating the mechanisms through which TFs have evolved and how they influence the evolution of other regulatory molecules can provide information on organism evolution and on the development of phenotypic variations. The aim of this review article was to provide a summary of the current literature on transcription factor evolution, function and how such evolution has played an important role in the emergence of complex organisms.
\end{abstract}

Correspondence to: Professor Dimitrios Vlachakis, Genetics and Computational Biology Group, Laboratory of Genetics, Department of Biotechnology, Agricultural University of Athens, 75 Iera Odos, 11855 Athens, Greece

E-mail: dimvl@aua.gr

Abbreviations: TF, transcription factor; PIC, pre-initiation complex; GTF, general transcription factor; CRE, cis-regulatory element; DBD, DNA binding domain; HD, homeodomain; HTH, helix-turn-helix; HMG, high mobility group; PTMs, post-translational modifications; CEBPB, CCAAT/enhancer binding protein beta; cAMP/PKA, cyclic AMP/protein kinase A; miRNAs, microRNAs; LBD, ligand-binding domain

Key words: transcription regulation, transcription factors, evolution, development, evo-devo, cis-regulatory elements, epigenetics

\section{Contents}
1. Introduction
2. Transcription factor evolution amongst life domains
3. cis-Regulatory elements
4. Co-operation among transcription factors
5. Transcriptional cofactors and post-translational modifications
6. Transcription factors and their expression patterns
7. Transcription factors and histone modifications
8. Transcription factors and miRNAs
9. Ligand-dependent transcription factors: The case of nuclear receptors
10. Conclusions

\section{Introduction}

Understanding evolution requires the elucidation of the mechanisms through which phenotypic variation is generated and its subsequent consequences. In early studies of genetics, genes were considered as trait-causing elements that are linearly arrayed on chromosomes (1). Extensive studies on developmental biology, though, have implied that a variety of elements control the actions of genes, and these actions can subsequently be altered $(2,3)$. It is now accepted that various elements regulate the actions of genes and, subsequently, phenotypic variation. Therefore, the manner in which gene information is expressed has become an important scientific topic. Gene expression is defined as a process through which the information encoded in a gene is used to direct the synthesis of a functional gene product (4). As a process, it may explain why organisms containing mostly the same DNA exhibit different cell types and functions (5). Gene expression is an intricate process and involves the coordination of multiple dynamic events, which are subject to multi-level regulation (6). Those regulatory levels include the transcriptional 
level, the post-transcriptional level, the translational level and the post-translational level. Regulating gene expression is crucial in living organisms (7). Gene regulation is essential in cellular differentiation in multicellular organisms, since it can contribute to the function and the structure of a specific cell, and is an integral part of organism development (4).

All of the above prove that apart from inherited genetic information, cell function and structure are influenced by information that is not encoded in the DNA sequence. This information has also been termed epigenetic information (5). Epigenetics is defined as both heritable alterations in gene activity and expression and also stable, long-term alterations in the transcriptional potential of a cell that may not be heritable (8). Epigenetics comprises of a number of mechanisms, which include DNA methylation, histone modification, post-translation modifications, chromatin remodeling and various forms of regulatory RNA molecules. These mechanisms seem to influence gene expression (5).

Gene transcriptional regulation is a fundamental part of both tissue-specific gene expression and gene activity in response to stimuli (9). The main regulators of gene transcription are transcription factors (TFs). TFs are defined as proteins that can bind specific DNA sequences to control transcription (10). Each cellular life form follows different strategies for the initiation and regulation of transcription. Prokaryotes use a single RNA polymerase, while eukaryotes exhibit multiple specialized RNA polymerases (11). Bacteria have two distinct mechanisms for the initiation of transcription, the promoter-centric mechanism, in which specific TFs interact with the promoter in order to alter its ability to bind RNA polymerase or RNA-centric mechanism, in which TFs interact with RNA in order to alter its promoter preference (12). In eukaryotes, a number of TFs interact with their cognate DNA motifs and recruit transcriptional cofactors to alter the chromatin environment. These TFs can also facilitate the assembly of a pre-initiation complex (PIC), which is composed of general TFs (GTFs) and RNA polymerase II (13). Lastly, the archaea transcriptional mechanism can be summarized as a simplified version of the eukaryotic transcriptional mechanism (14). Archaea feature a transcriptional apparatus that includes additional RNA polymerase subunits and basal TFs that direct transcription initiation and elongation. TFs in the case of archaea recruit the RNA polymerase to the specific DNA domain.

The above underline the importance of TFs in both the initiation and regulation of gene transcription. The activation of TFs is quite complex and may involve multiple intracellular transduction pathways or direct activation through specific molecules that bind, known as ligands (15). TFs mostly regulate gene activity by binding to specific short DNA base pair patterns termed motifs or cis-regulatory elements (CREs) in upstream, intron, or downstream regions of target genes. They can also act by interacting with other genomic locations that may be distant to the primary DNA sequence (16). These are defined as gene regulatory regions. (17) CREs include promoters and sequences called enhancers in cases of transcriptional activation and silencers in cases of transcriptional repression (18). The specific domain TFs have that can bind DNA is termed DNA binding domain (DBD). TFs use a variety of DNA-binding structural motifs to recognize their target sequences, which include homeodomain (HD), helix-turn-helix (HTH) and high-mobility group box (HMG). Such DBDs can be used to classify TFs. The interaction between DNA and TFs goes beyond the structural and sequence level since several other factors participate in the process, such as the influence of cofactors, epigenetic modifications and the cooperative binding of other TFs (19). Thus, gene regulation involves a large number of molecular mechanisms. Therefore, an in-depth examination of the evolution of TFs, which takes into account the interaction with all the molecular factors mentioned above, and the manner through which TFs influence the evolution of other molecular mediators, is essential to the understanding of organism evolution.

\section{Transcription factor evolution amongst life domains}

TF function involves two basic features: i) The ability to recognize and bind short, specific sequences of DNA within regulatory regions; and ii) the ability to recruit or bind proteins that participate in transcriptional regulation (20). Consequently, the evolution of TFs mainly depends on alterations in binding sites, binding partners and expression patterns (10). Moreover, as an integral part of gene expression, they are closely related to the evolution of epigenetic mechanisms (5). The current literature on TF evolution provides a broad range of information. Firstly, gene duplication and gene loss as crucial drivers of evolution $(21,22)$ are subsequently important drivers of TF evolution. Regardless of organism complexity, they are present in all domains of life. Duplication and deletion can influence transcriptional regulatory networks by increasing or reducing the number of TFs with specific binding preferences $(23,24)$. Following the duplication of a TF gene, the two resulting gene copies are likely the same. Since they share the same sequence, including the DBD sequence, they bind to the same target genes. Ensuing mutations in the DNA binding domain sequence can lead to one of the TF copies to switch to regulating different target genes. On a more lineage-specific level, TFs display several differences. Although the basal transcription machinery has long been considered universally conserved, it is currently accepted that it too diversifies during evolution. The size and subunit composition of the basal transcription machinery increase highly during evolution, consisting of roughly 6 subunits in bacteria, up to 15 in the archaea, and a large number in eukaryotes, which have at least 3 different RNA polymerases (25). Significant differences are apparent between prokaryotes and eukaryotes. Firstly, some DBDs are specific to evolutionary lineages; e.g., the ribbon-helix-helix domain is specific to bacteria and archaea while $\mathrm{C} 2 \mathrm{H} 2-\mathrm{ZNfs}$, Homeobox box, and T-box domains are specific to eukaryotes (26). Moreover, eukaryotic TFs are relatively longer than other eukaryotic proteins with a different function, while this association is reversed in prokaryotes. This phenomenon may be due to the fact that eukaryotic TFs have a number of long intrinsic disordered segments that are needed to leverage the formation of a multi-protein transcription protein complex (27). Another characteristic specific to eukaryotes are the repeats of the same DBD family in one polypeptide chain. This characteristic may be the result of a mechanism eukaryotes use that increases the length and diversity of DNA binding recognition sequences using a limited number of DNA binding domain families (27). 


\section{3. cis-Regulatory elements}

Changes in CREs may influence TF evolution and function and vice versa $(28,29)$. TFs can bind a single DNA binding site or full promoter/enhancer/silencer regions that feature multiple binding sites. Several factors seem to affect the evolution, emergence, disappearance and function of CREs. These factors include insertion and deletion mutational mechanisms, slippage processes, tje large rearrangement of promoter regions, co-operation amongst TFs and the existence of initial sequence distributions that are biased towards the mutational neighborhood of strongly binding sequences $(30,31)$. Insertion and deletion mutational mechanisms can lead to the slow emergence of binding sites out of a random sequence, while factors that accelerate these processes may include the already sufficient genomic sequence from which sites can evolve and the possible co-operativity between adjacent TFs (30). Furthermore, since the interaction of TFs' with TF binding sites is integral in gene regulation, a mutation in either $\mathrm{TF}$ or binding site hinders that interaction and may lead to dysfunctional gene expression. Therefore, in order to maintain proper gene expression levels, TF evolution and CREs evolution are closely intertwined (32). They specifically bear a co-evolutionary association, where in order to sustain proper interaction, a mutation in one interacting partner could be compensated by a corresponding mutation in its' interacting partner during the course of evolution (32).

\section{Co-operation among transcription factors}

Although prokaryote individual TFs can recognize long DNA motifs that are alone capable of defining the genes they may regulate, organisms with larger genomes are characterized by TFs that recognize sequences too short to be able to define unique genomic positions. Moreover, the development of multicellular organisms requires molecular systems that are complex and able to execute combinational processes. In an effort to overcome these obstacles, organisms have evolutionary developed co-operative recognition of DNA by multiple TFs (33). TFs can collaborate through a variety of mechanisms, with each co-operative mechanism determining the specifics of the regulatory interaction. Some of the mechanisms through which TFs cooperate include protein-protein interaction and indirect co-operation (33). A prime example of protein-protein interaction among TFs is the formation of functional dimers. A number of eukaryotic TFs proteins are not able to bind DNA sequences as monomeric proteins and require physical interaction with an identical molecule or one within the same family to form functional dimers that are able to bind targeted DNA sequences. It has been suggested that, at first, TFs function as monomers, something supported by the fact that TFs in less complex organisms can sufficiently bind target sequences as monomers (34). Several promoters that include symmetrical palindromic repeats of the DNA-recognition motif could have potentially brought two or more copies of the same TF protein into proximity. If, by chance, an interaction domain with only one interaction sequence appeared, then this would help establish the formation of a TF complex on DNA because this specific complex would recognize a larger DNA motif (34). These events would lead to more relaxed evolutionary constraints on the TF DBD within a redundant duplicate gene and would allow the emergence of a DNA-binding domain that binds with less affinity, but is still functional. Once such evolutionary steps are taken, the TF must function as an obligate dimer. Consequently, further duplication and changes in specificity gave rise to the appearance and diversification of the various TFs' dimerizing families (34). The Co-operative binding of TFs to DNA can also occur without direct protein-protein interactions. This co-operation is achieved through a process known as indirect co-operativity or collaborative competition, in which a cohort of TFs collectively competes with the same histone octamer for access to the underlying DNA (35). Collaborative competition arises automatically from the close juxtaposition of binding sites for arbitrarily chosen TFs (36). Therefore, collaborative competition may play an important role in the evolution of gene regulatory modules, since molecules that undergo combinatorial regulation may be assembled from randomly selected components, with no requirement for coevolution. Following the coevolution of the required partners can increase the co-operativity through the aforementioned protein-protein contacts or bridging proteins, and may thus increase the magnitude of combinational control (36).

\section{Transcriptional cofactors and post-translational modifications}

A number of co-activator and co-repressor proteins are components of multi-subunit coregulator complexes that exhibit diverse enzymatic activities (37). Specifically, TF activity is regulated via post-translational modifications (PTMs) by such modification enzymes as a response to cellular stimuli (38). Modification enzymes directly interact with TFs and modify specific residues of the TF protein and alter subcellular localization, stability, interaction with more cofactors and other transcriptional activities (39). Some of the modifications those enzymes undertake are phosphorylation, acetylation, methylation and glycosylation (38). It is, therefore, likely that PTMs of histones, TFs, or polymerase II and its associated proteins at the PIC are involved in enhancer-core-promoter communication and potentially, in the combinatorial regulation of transcription activation (40). Thus, it is not unexpected that recent research has demonstrated connections between novel PTMs sites within TFs and the evolution of new features (20). A prime example is the evolution of pregnancy in mammals, in which amino-acid changes in the TFCCAAT/enhancer-binding protein beta (CEBPB) change the manner in which it responds to cyclic AMP/protein kinase A (cAMP/PKA) signaling (41). Such amino-acid changes reorganize the location of key phosphorylation sites and may change the response of CEBPB to phosphorylation from repression to activation (41).

\section{Transcription factors and their expression patterns}

TFs in eukaryotes are functionally divergent between different species and paralogs, which proves that they can evolve new functions (42). In eukaryotes, 5 groups of TFs with distinct expression patterns have emerged through periodic expansion in the TF repertoire. These include groups that are present only in primates, those that are generally found in mammals, 
vertebrates, or metazoan and those found in most eukaryotes, including yeast (43). A mechanism that can drive such evolutionary alterations in TF function is tissue-specific gene expression (42). Tissue-specific gene expression as a mechanism can enhance the specificity of TFs through minimizing the pleiotropic effects of mutations that could lead to the gain of novel regulatory links via TF evolution, while simultaneously restricting the effects of loss of function mutations that break important regulatory links (42). A more thorough understanding of the association between TFs and tissue specificity can be achieved by studying the expansions that occur in the TF repertoire in conjunction with the evolution of tissue-specific mechanisms. The expansions mentioned above seem to have occurred unevenly for TFs containing different types of DNA-binding domains. Some DBDs have expanded rapidly through evolution, while others have not expanded significantly since their emergence. These expansions could have provided evolution with the means through which to modify or create different expression patterns for transcriptional factors, including tissue-specific ones, by duplication followed by promoter divergence (43).

\section{Transcription factors and histone modifications}

Human cell DNA is wrapped around histone protein octamers. This protein complex is known as the 'nucleosomal core particle' (44). Histone proteins have tails which include residues that can be post-translationally modified and influence transcription. This effect on transcription is regulated by changes in histone modification patterns that surround TF binding motifs (45). It is speculated that TFs with evolutionary related DNA binding domains sample putative binding sites with similar histone modification pattern environments (45).

\section{Transcription factors and miRNAs}

MicroRNAs (miRNAs or miRs) are small regulatory non-coding RNAs that influence gene regulation (46). Along with TFs, they play an important role in gene regulatory network evolution (47). It seems that gene expression is mainly regulated by TFs at the transcriptional level and miRNA at the post-transcriptional level, with both expression regulators having the ability to regulate each other. The interplay between TFs and miRNAs provides specific constraints and innovations for the evolution of such networks. It is thus expected that they also exhibit some form of coevolution. Coevolution seems to exist in TF and miRNAs pairs that are connected by transcriptional activation signals but not in pairs that are connected by transcriptional repression signals (48). This association may be explained by the fact that TFs that trigger miRNA expression can subsequently function together with the activated miRNAs, while TFs that repress the expression of specific miRNAs will not function with them later and thus be under their evolutionary influence (48).

\section{Ligand-dependent transcription factors: The case of nuclear receptors}

As it has been already stated, a number of transcriptional factors may be activated through ligand binding. In such cases, ligands are essential to TF function and are expected to be an integral part of their evolution. Prime examples of ligand-dependent TFs are nuclear receptors (NRs), which modulate gene transcription in direct response to small lipophilic molecules (49). Apart from the characteristic DBD of all TFs, NRs feature a structural domain that binds ligands, termed ligand-binding domain (LBD). Ligand binding induces a conformational change that activates the receptor and stimulates the activity of its' target gene (50). NR ligands are product intermediates of various metabolic pathways. This fact means that they have been evolutionary established through genetic modulations on the components of particular metabolic pathways but not through modification of a single gene. Consequently, this fact indicates that NR evolution is heavily influenced not only by selective genes but by the ever-expanding interaction among gene networks (51).

\section{Conclusions}

Studying the evolution of developmental processes is essential in shedding light on how morphological diversity emerged (52). TFs have a major role in multicellular eukaryotes development, and they are the main regulators of embryonic development in embryophytes and metazoans (53). This role is expected since development is controlled mainly by gene regulatory networks, which in turn are controlled by TFs (52). TFs appear to regulate up to hundreds of genes that are related to organism development and hint at the existence of vast transcription-factor regulated networks. These networks include a large number of components. The evolution of these networks, which includes both TFs and their components, provides a snapshot of how complex organisms emerged and a basis for the study of evolutionary developmental biology.

\section{Acknowledgements}

Not applicable.

\section{Funding}

DV would like to acknowledge funding from: i) Microsoft Azure for Genomics Research Grant (CRM:0740983); ii) FrailSafe Project (H2020-PHC-21-2015 - 690140) 'Sensing and predictive treatment of frailty and associated co-morbidities using advanced personalized models and advanced interventions', co-funded by the European Commission under the Horizon 2020 research and innovation program; iii) Amazon Web Services Cloud for Genomics Research Grant (309211522729); iv) AdjustEBOVGP-Dx (RIA2018EF-2081): Biochemical Adjustments of native EBOV Glycoprotein in Patient Sample to Unmask target Epitopes for Rapid Diagnostic Testing. A European and Developing Countries Clinical Trials Partnership (EDCTP2) under the Horizon 2020 'Research and Innovation Actions' DESCA. EE would like to acknowledge funding by the project 'INSPIRED-The National Research Infrastructures on Integrated Structural Biology, Drug Screening Efforts and Drug Target Functional Characterization' (Grant MIS 5002550) and by the project: 'OPENSCREEN-GR An Open-Access Research Infrastructure of Chemical Biology and Target-Based Screening Technologies for Human and Animal Health, Agriculture and 
the Environment' (Grant MIS 5002691), which are implemented under the Action 'Reinforcement of the Research and Innovation Infrastructure', funded by the Operational Programme 'Competitiveness, Entrepreneurship and Innovation' (NSRF 2014-2020) and co-financed by Greece and the European Union (European Regional Development Fund).

\section{Availability of data and materials}

Not applicable.

\section{Authors' contributions}

TM, AE, FB, DV, GPC, EE have all equally contributed to the writing, drafting, revising, editing, reviewing, and the conception and design of the study. All authors have read and approved the final manuscript.

\section{Ethics approval and consent to participate}

Not applicable.

\section{Patient consent for publication}

Not applicable.

\section{Competing interests}

The authors declare that they have no competing interests.

\section{References}

1. Emerson $\mathrm{JJ}$ and Li WH: The genetic basis of evolutionary change in gene expression levels. Philos Trans R Soc Lond B Bio Sci 365: 2581-2590, 2010.

2. Breidbach $\mathrm{O}$ and Ghiselin MT: Evolution and development: past, present, and future. Theory Biosci 125: 157-171, 2007.

3. Reilly SK and Noonan JP: Evolution of Gene Regulation in Humans. Annu Rev Genomics Hum Genet 17: 45-67, 2016.

4. García-Sánchez A and Marqués-García F: Review of Methods to Study Gene Expression Regulation Applied to Asthma. Methods Mol Biol 1434: 71-89, 2016.

5. Gibney ER and Nolan CM: Epigenetics and gene expression. Heredity 105: 4-13, 2010.

6. Preziosi P: 2.06-Drug Development. In: Comprehensive Medicinal Chemistry II. Taylor JB and Triggle DJ (eds). Elsevier, Oxford, pp173-202, 2007.

7. Atkinson TJ and Halfon MS: Regulation of gene expression in the genomic context. Comput Struct Biotechnol J 9: e201401001, 2014

8. Laker RC and Ryall JG: DNA Methylation in Skeletal Muscle Stem Cell Specification, Proliferation, and Differentiation. Stem Cells Int 2016: 5725927, 2016.

9. Latchman DS: Transcription factors: An overview. Int J Biochem Cell Biol 29: 1305-1312, 1997.

10. Lambert SA, Jolma A, Campitelli LF, Das PK, Yin Y, Albu M, Chen X, Taipale J, Hughes TR and Weirauch MT: The Human Transcription Factors. Cell 172: 650-665, 2018.

11. Klenk H-P and Doolittle WF: Evolution. Archaea and eukaryotes versus bacteria? Curr Biol 4: 920-922, 1994.

12. Lee DJ, Minchin SD and Busby SJ: Activating transcription in bacteria. Annu Rev Microbiol 66: 125-152, 2012.

13. Venters BJ and Pugh BF: How eukaryotic genes are transcribed. Crit Rev Biochem Mol Biol 44: 117-141, 2009.

14. Gehring AM, Walker JE and Santangelo TJ: Transcription Regulation in Archaea. J Bacteriol 198: 1906-1917, 2016.

15. Adcock IM and Caramori G: Transcription Factors. In: Asthma and COPD. Barnes PJ, Drazen JM, Rennard SI and Thomson NC (eds). 2nd edition. Academic Press, Oxford, pp373-380, 2009.
16. Powell RV, Willett CR, Goertzen LR and Rashotte AM: Lineage specific conservation of cis-regulatory elements in Cytokinin Response Factors. Sci Rep 9: 13387, 2019.

17. Wilkinson AC, Nakauchi $H$ and Göttgens B: Mammalian Transcription Factor Networks: Recent Advances in Interrogating Biological Complexity. Cell Syst 5: 319-331, 2017.

18. Rebeiz $\mathrm{M}$ and Tsiantis $\mathrm{M}$ : Enhancer evolution and the origins of morphological novelty. Curr Opin Genet Dev 45: 115-123, 2017.

19. Yesudhas D, Batool M, Anwar MA, Panneerselvam S and Choi S: Proteins Recognizing DNA: Structural Uniqueness and Versatility of DNA-Binding Domains in Stem Cell Transcription Factors. Genes (Basel) 8: E192, 2017.

20. Cheatle Jarvela AM and Hinman VF: Evolution of transcription factor function as a mechanism for changing metazoan developmental gene regulatory networks. Evodevo 6: 3, 2015.

21. Zhaxybayeva O, Lapierre P and Gogarten JP: Ancient gene duplications and the $\operatorname{root}(\mathrm{s})$ of the tree of life. Protoplasma 227 : 53-64, 2005.

22. Albalat R and Cañestro C: Evolution by gene loss. Nat Rev Genet 17: 379-391, 2016

23. Rosanova A, Colliva A, Osella M and Caselle M: Modelling the evolution of transcription factor binding preferences in complex eukaryotes. Sci Rep 7: 7596, 2017.

24. Pérez-Rueda E, Collado-Vides J and Segovia L: Phylogenetic distribution of DNA-binding transcription factors in bacteria and archaea. Comput Biol Chem 28: 341-350, 2004.

25. Duttke SH: Evolution and diversification of the basal transcription machinery. Trends Biochem Sci 40: 127-129, 2015.

26. Nowick K and Stubbs L: Lineage-specific transcription factors and the evolution of gene regulatory networks. Brief Funct Genomics 9: 65-78, 2010.

27. Charoensawan V, Wilson D and Teichmann SA: Genomic repertoires of DNA-binding transcription factors across the tree of life. Nucleic Acids Res 38: 7364-7377, 2010.

28. Inukai S, Kock KH and Bulyk ML: Transcription factor-DNA binding: Beyond binding site motifs. Curr Opin Genet Dev 43: 110-119, 2017.

29. Siepel A and Arbiza L: Cis-regulatory elements and human evolution. Curr Opin Genet Dev 29: 81-89, 2014.

30. Tuğrul M, Paixão T, Barton NH and Tkačik G: Dynamics of Transcription Factor Binding Site Evolution. PLoS Genet 11: e1005639, 2015.

31. Berg J, Willmann S and Lässig M: Adaptive evolution of transcription factor binding sites. BMC Evol Biol 4: 42, 2004.

32. Yang S, Yalamanchili HK, Li X, Yao KM, Sham PC, Zhang MQ and Wang $\mathrm{J}$ : Correlated evolution of transcription factors and their binding sites. Bioinformatics 27: 2972-2978, 2011

33. Morgunova E and Taipale J: Structural perspective of cooperative transcription factor binding. Curr Opin Struct Biol 47: 1-8, 2017.

34. Amoutzias GD, Robertson DL, Van de Peer Y and Oliver SG Choose your partners: Dimerization in eukaryotic transcription factors. Trends Biochem Sci 33: 220-229, 2008.

35. Long HK, Prescott SL and Wysocka J: Ever-Changing Landscapes: Transcriptional Enhancers in Development and Evolution. Cell 167: 1170-1187, 2016.

36. Miller JA and Widom J: Collaborative competition mechanism for gene activation in vivo. Mol Cell Biol 23: 1623-1632, 2003.

37. Rosenfeld MG, Lunyak VV and Glass CK: Sensors and signals: A coactivator/corepressor/epigenetic code for integrating signal-dependent programs of transcriptional response. Genes Dev 20: 1405-1428, 2006.

38. Everett L, Hansen M and Hannenhalli S: Regulating the regulators: Modulators of transcription factor activity. Methods Mol Biol 674: 297-312, 2010.

39. Tootle TL and Rebay I: Post-translational modifications influence transcription factor activity: A view from the ETS superfamily. BioEssays 27: 285-298, 2005.

40. Reiter F, Wienerroither S and Stark A: Combinatorial function of transcription factors and cofactors. Curr Opin Genet Dev 43: 73-81, 2017

41. Lynch VJ, May G and Wagner GP: Regulatory evolution through divergence of a phosphoswitch in the transcription factor CEBPB. Nature 480: 383-386, 2011.

42. Lynch VJ and Wagner GP: Resurrecting the role of transcription factor change in developmental evolution. Evolution 62: 2131-2154, 2008.

43. Vaquerizas JM, Kummerfeld SK, Teichmann SA and Luscombe NM: A census of human transcription factors: Function, expression and evolution. Nat Rev Genet 10: 252-263, 2009. 
44. Lawrence M, Daujat S and Schneider R: Lateral Thinking: How Histone Modifications Regulate Gene Expression. Trends Genet 32: 42-56, 2016.

45. Xin B and Rohs R: Relationship between histone modifications and transcription factor binding is protein family specific. Genome Res 28: 321-333, 2018.

46. O'Brien J, Hayder H, Zayed Y and Peng C: Overview of MicroRNA Biogenesis, Mechanisms of Actions, and Circulation. Front Endocrinol (Lausanne) 9: 402, 2018.

47. Chen $\mathrm{K}$ and Rajewsky N: The evolution of gene regulation by transcription factors and microRNAs. Nat Rev Genet 8: 93-103, 2007.

48. Qiu C, Wang J, Yao P, Wang E and Cui Q: microRNA evolution in a human transcription factor and microRNA regulatory network. BMC Syst Biol 4: 90, 2010

49. Rachez C, Lemon BD, Suldan Z, Bromleigh V, Gamble M, Näär AM, Erdjument-Bromage H, Tempst $P$ and Freedman LP: Ligand-dependent transcription activation by nuclear receptors requires the DRIP complex. Nature 398: 824-828, 1999.
50. Sharma Y, Chilamakuri CS, Bakke $M$ and Lenhard B: Computational characterization of modes of transcriptional regulation of nuclear receptor genes. PLoS One 9: e88880, 2014

51. Holzer G, Markov GV and Laudet V: Evolution of Nuclear Receptors and Ligand Signaling: Toward a Soft Key-Lock Model? Curr Top Dev Biol 125: 1-38, 2017.

52. Carroll SB: Evo-devo and an expanding evolutionary synthesis: A genetic theory of morphological evolution. Cell 134: 25-36, 2008.

53. de Mendoza A, Sebé-Pedrós A, Šestak MS, Matejcic M, Torruella G, Domazet-Loso T and Ruiz-Trillo I: Transcription factor evolution in eukaryotes and the assembly of the regulatory toolkit in multicellular lineages. Proc Natl Acad Sci USA 110: E4858-E4866, 2013.

This work is licensed under a Creative Commons Attribution-NonCommercial-NoDerivatives 4.0 International (CC BY-NC-ND 4.0) License. 